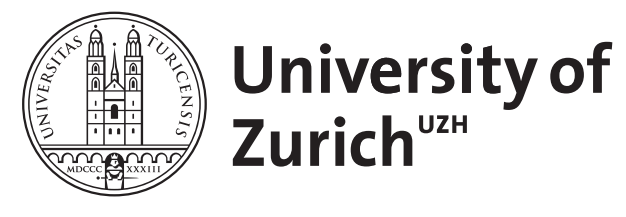

\title{
Ist Sauerstoffmangel immer nur schädlich?
}

Schläpfer, Martin

\begin{abstract}
Die Aufgabe des Kreislaufs ist die Versorgung von Gewebe mit Sauerstoff und Nährstoffen. Zustände des Sauerstoffmangels (Hypoxie) werden als bedrohlich erachtet, da abhängig vom Ausprägungsgrad Zellen absterben, sei es durch apoptotische oder nekrotische Vorgänge. Dem versucht das Gewebe durch evolutionär erhaltene Signalwege entgegenzuwirken, z.B. über den nukleären Hypoxie-induzierbaren Faktor, der das Gewebe schützen soll, indem er das Überleben der Zellen fördert und gleichzeitig in Angiogenese, Hämatogenese und Stoffwechselprozesse eingreift. Neuere Erkenntnisse weisen darauf hin, dass gerade diese konservierten Signalwege auch therapeutische Ansätze in der Wundheilung von Knochen und Haut sowie in der Regeneration von Geweben, z.B. der Leber, und dem hämatopoetischen System, bedeuten können.
\end{abstract}

DOI: https://doi.org/10.1024/1661-8157/a003070

Posted at the Zurich Open Repository and Archive, University of Zurich ZORA URL: https://doi.org/10.5167/uzh-158381

Journal Article

Accepted Version

Originally published at:

Schläpfer, Martin (2018). Ist Sauerstoffmangel immer nur schädlich? Praxis, 107(21):1155-1159.

DOI: https://doi.org/10.1024/1661-8157/a003070 
Ist Sauerstoffmangel immer nur schädlich?

Martin Schläpfer

Institut für Anästhesiologie und Physiologie, UniversitätsSpital und Universität Zürich

Schlüsselwörter: Sauerstoffmangel, Hyposie-induzierbarer Faktor, Regeneration

Korrespondenzadresse:

PD Dr. med. Martin Schläpfer, MSc

Institut für Anästhesiologie

UniversitätsSpital Zürich

Rämistrasse 100

8091 Zürich, Schweiz

Mail: martin.schlaepfer@usz.ch 


\section{Zusammenfassung}

Die Aufgabe des Kreislaufs ist die Versorgung des Gewebes mit Sauerstoff und Nährstoffen. Zustände des Sauerstoffmangels (Hypoxie) werden als bedrohlich erachtet, da abhängig vom Ausprägungsgrad Zellen absterben, sei es durch apoptotische oder nekrotische Vorgänge. Dem versucht das Gewebe durch evolutionär erhaltene Signalwege entgegenzuwirken, so über den nukleären Hypoxie-induzierbaren Faktor (HIF), der das Gewebe schützen soll, indem er das Überleben der Zellen fördert und gleichzeitig in Angiogenese, Hämatogenese und Stoffwechselprozesse eingreift. Neuere Erkenntnisse weisen darauf hin, dass gerade diese konservierten Signalwege auch therapeutische Ansätze in der Wundheilung von Knochen und Haut, sowie der Regeneration von Geweben, beispielsweise der Leber und dem hämatopoetischen System, bedeuten können.

\section{Hintergrund}

Die Versorgung aller Gewebe mit Nahrung und Sauerstoff ist die zentrale Aufgabe des Kreislaufs. Die Themen Kreislauf, Sauer- und Nährstoffversorgung des Körpers sind deshalb seit Jahren zentraler Gegenstand vieler Forschungsarbeiten. In heute historischen Publikationen wurde festgestellt, dass unterschiedliche Gewebe des menschlichen Körpers unterschiedlich viel Sauerstoff verbrauchen. Das Gehirn benötig beispielsweise $10 \mathrm{ml}$ [1], das Herz $8 \mathrm{ml}$ [2] und Fettgewebe 2ml [3] Sauerstoff pro Gramm Gewebe und Minute. Aus diesen Fakten lässt sich ableiten, dass der Sauerstoffmangel nicht durch eine Zahl, sondern durch den Bedarf des jeweiligen Gewebetyps definiert ist. Ein Sauerstoffmangel innerhalb einer Zelle wird durch den Abfall des Sauerstoffpartialdrucks festgestellt [4]. Da maligne entartete Gewebe in der Regel schneller wachsen als deren versorgende Gefässe, entstehet in 
bösartigen Tumoren oft ein Sauerstoffgradient [5]. Viele Erkenntnisse rund um die Hypoxieforschung stammen somit aus der Krebsforschung.

\section{Sauerstoffmangel - the ugly!}

\section{Sauerstoffmangel und ATP-Produktion}

Tritt in einem Gewebe akut ein nahezu oder ein vollständiger Sauerstoffmangel auf, erliegt die Energieproduktion, also die Synthese von Adenosin-Triphosphat (ATP). Die $\mathrm{Na}^{+} / \mathrm{K}^{+}-$ ATPase kann somit ihrer Funktion, dem Aufrechterhalten des zellulären Elektrolytgleichgewichts, nicht mehr nachkommen. Der Transport von lonen und Wasser ist essentiell für die Homöostase der Zelle, nur bei erhaltener lonen- uns Wasserverteilung kann die Zelle überleben.

\section{Sauerstoffmangel und Zelltod}

Auf den sistierenden lonentransport folgt eine Zellschwellung, eine Umverteilung der Ionenkonzentration und schliesslich der Zelluntergang. Das Bild des Zelltodes nach dem Erliegen der ATP Produktion entspricht dem Bild einer Nekrose [6]. Die Nekrose, charakterisiert durch Schwellung, Verlust der Membranintegrität und Zelllyse, geht mit einer entzündlichen Reaktion einher. Die Apoptose stellt demgegenüber einen programmierten Zelltod dar, der charakterisiert wird von Zellschrumpfung, Chromatinkondensation, dem Auftreten von Vakuolen und der Bildung apoptotischer Zellfragmente; bei deren Phagozytose bleibt in der Regel eine Entzündungsreaktion aus. Eine Hypoxie kann sowohl die Nekrose [7] oder die Apoptose [8] auslösen, die Nekrose ist aber schwerem Sauerstoffmangel vorherrschend. 


\section{Sauerstoffmangel - reducing the bad}

\section{Der HIF-Pathway}

Das Erkennen der zellulären Hypoxie geschieht über den Hypoxie-induzierbaren Faktor (HIF), einen hochkonservierten Transkriptionsfaktor, der in Abhängigkeit vom zellulärem Sauerstoff Signalkaskaden in Gang bringt und verschiedene Zielgene regulieren kann. Bei der HIF-Kaskade, dem momentan wahrscheinlich am besten beschriebenen Hypoxiesensor der Zelle stellt nicht HIF selbst, sondern vielmehr die Prolylhydroxylase (PHD) den eigentlichen Sauerstoffsensor dar [9]. Es existieren vier verschiedene PHD Isoformen (PHD1-4). Die Isoformen PHD1-3 greifen Sauerstoffpartialdruck-abhängig in den HIF-Pathway ein, während die Rolle von PHD4, einem mitochondrialen Transmembranprotein, noch nicht abschliessend geklärt ist [10]. Es ist aber bekannt, dass PHD4 auch HIF-unabhängig in die Angiogenese bei Malignomen eingreifen kann [11]. Das HIF-Heterodimer besteht in seiner aktiven Form aus einer $\alpha$-Untereinheit und einer $\beta$-Untereinheit. Unter normoxischen Bedingungen verwenden Prolylhydroxylasen (PHD) Sauerstoff, um zwei Prolin-Reste von HIF $\alpha$ zu hydroxylieren; dadurch werden diese vom von Hippel Lindau (VHL) Protein erkannt, in einem mehrstufigen Prozess ubiquitiniert, und schliesslich durch proteasomale Degradation abgebaut $[12,13]$. Fehlt der Zelle Sauerstoff, ist PHD inaktiv. Die Hydroxylierungsreaktion findet nicht mehr statt und HIF bleibt stabil (Figur 1). So kann HIF als Transkriptionsfaktor an das Hypoxia Response Element (HRE) in der Promotorregion seiner Zielgene binden und diese aktivieren. Von HIF $\alpha$ existieren zwei Isoformen, die im Körper teilweise unterschiedliche Effekte ausüben, aber zusammen die HIF-vermittelte Hypoxie-Antwort auslösen [14]. Wie bereits erwähnt ist weniger ein absoluter Sauerstoffwert, als vielmehr die Veränderung der Sauerstoffspannung, die Ursache für die Stabilisierung von HIF [4]; folglich 
haben unterschiedliche Zellen unterschiedliche Schwellenwerte für einen Sauerstoffmangel. Neben den unterschiedlichen Schwellenwerten wurde ebenfalls, eine gewebeabhängig unterschiedliche HIF Kinetik beschrieben [15], also ein unterschiedlich schnelles Erreichen der maximalen HIF Konzentration. Eine Aktivierung der HIF-Signalkaskade greift in zentrale Mechanismen ein: das Zellüberleben, die Apoptose, die Angiogenese, die Blutbildung und die Metabolik [16].

\section{Zellzyklus und Zellüberleben}

Um im proliferierenden Geweben den Sauerstoffverbrauch zu reduzieren, können Zellen durch Aktivierung des HIF-Pathways am Übergang der G1/S-Phase in den Zellarrest gebracht werden[17]. Dies verhindert eine energiekonsumierende Zellteilung. Weiter kann HIF in die Apoptose eingreifen. Diese Effekte wurden vor allem in der Forschung rund um Malignome etabliert. HIF kann zum Zellüberleben beitragen, aber auch die Apoptose auslösen. Die pround anti-apoptotischen Effekte werden zumindest teilweise durch die Existenz von drei unterschiedlichen $\alpha$-Untereinheiten erklärt [18].

\section{Verbesserung der Sauerstoffversorgung durch Hämatopoese und Angiogenese}

Bevor HIF als hochkonservierter Transkriptionsfaktor bei Sauerstoffmangelzuständen beschrieben wurde, wurde er als Erythropoietin-Produktion induzierender Faktor in der Niere identifiziert[19, 20]. Erythropoietin seinerseits stimuliert hämatogene Stammzellen, was in der Erythropoese, also der Bildung roter Blutzellen resultiert[21]. Beim Abfall der Sauerstoffspannung im Nierengewebe versucht der Körper die Sauerstoffversorgung durch die Produktion von Erythrozyten auszugleichen. Vor dieser Tatsache erscheint es logisch, dass HIF auch in die Angiogenese eingreift und diese anregt. Durch die Stabilisierung von HIF 
werden verschiedene pro-angiogenetische Faktoren induziert: Vascular endothelial growth factor (VEGF), Angiopoetin, aber auch pro-angiogenetische Rezeptoren wie zum Beispiel der VEGF-R[22] werden vermehrt gebildet. Auch diese Effekte wurden vor allem bei Malignomen untersucht, wo durch die rasch wachsende Zellmasse eine zentrale Hypoxie entsteht und die Angiogenese durch die beschriebenen Mechanismen gefördert wird [23]. Über die zusätzliche Produktion von induzierbarer NO-Synthetase (iNOS) wird NO freigesetzt, was mittels Vasodilatation und Unterstützung der Angiogenese [24] die Sauerstoffversorgung begünstigt.

Anpassung der Stoffwechsellage: Bei Hypoxie fehlt dem Gewebe der Sauerstoff für die Ausübung der aeroben Glycolyse, es erfolgt die anaerobe Glycolyse. Dadurch werden pro Mol Glucose nur noch 2 anstelle von 36 Mol ATP gebildet. Dieselbe Menge an Energie benötigt folglich mehr Glucose. Die Stabilisierung von HIF begünstigt die Glycolyse; zudem wird die Produktion von Glucosetransportern (GLUT1) aufreguliert [25], was den Glucosetransport zum hypoxischen Gewebe begünstigt. Der Lactatanfall, einhergehend mit der anaeroben Glycolyse, führt zur lokalen Azidose. Zu deren Reduktion wird die Transkription der Carboanhydrase-9 durch HIF induziert [26, 27].

\section{Sauerstoffmangel - promoting the good?}

Während wir Sauerstoffmangel in der Regel mit etwas Schlechtem assoziieren, kann ein kurzzeitiger oder mässiger Sauerstoffmangel, respektive die Aktivierung von Signalkaskaden nach Sauerstoffmangel therapeutische Optionen anbieten! 
Der erste Schutzmechanismus zur Verminderung eines Ischämieschadens wurde im Rahmen der ischämischen Präkonditionierung in den 80er Jahren beschrieben. Im experimentellen Ansatz hat man festgestellt, dass ein kurzzeitiger Verschluss einer Koronararterie vor $\mathbf{4 0}$ minütiger Ischämie die kardiale Nekrose gegenüber einer einmaligen 40 minütigen Ischämie reduziert [28]. Dieses Phänomen konnte in diversen Organen, zum Beispiel auch dem Gehirn bestätigt werden [29]. Neueren Datums ist die Erkenntnis, dass im Rahmen des «Remote Ischemic Preconditionings» die erste kürzere Ischämie durchaus am Arm - also einem dem eigentlichen Schaden entfernten Gebiet - stattfinden kann und in der Herzchirurgie trotzdem den Ischämieschaden des Myokards verringert [30]. Es ist jedoch hervorzuheben, dass sowohl die ischämische Präkonditionierung, als auch das «Remote Ischemic Preconditioning» nicht HIF-vermittelte Effekte sind. Während bei der ischämischen Präkonditionierung die Aktivierung der Proteinkinase C zentral zu sein scheint [31, 32], werden beim «Remote Ischemic Preconditioning» zum aktuellen Zeitpunkt verschiedene Substanzen als Vermittler diskutiert, unter anderem Adenosin, Endocannabionide, Bradykinin, Opioide, Mikrovesikel, Apolipoprotein A-I und Nitrite [33].

\section{Verbesserung der Regeneration}

Ebenso bemerkenswert ist, dass nach Knochenfraktur die Stabilisierung von HIF beobachtet wird. Diese verbessert über VEGF die Vaskularisierung und trägt zur Knochenheilung bei [34]. In Analogie dazu wurde bei der Verletzung der Haut die Stabilisierung von HIF beobachtet [35]. Während die Stabilisierung von HIF einerseits zur Wundheilung der Haut beiträgt, resultiert eine langfristige Hypoxie in einer Wundheilungsstörung [36].

\section{Beschleunigung der Regeneration}


In der Leber führt eine partielle Hepatektomie, aber auch eine selektive Ligation von Portalgefässen zur Geweberegeneration [37]. Diese wird durch die Stabilisierung von HIF, etwa mittels PHD-Inhibitoren experimentell beschleunigt [38]. Möglicherweise kann durch diese Massnahme in Zukunft bei Patienten mit aktuell nicht resezierbaren Malignomen in der Leber, gesundes Leberparenchym selektiv zur Regeneration angeregt werden. Dies würde ermöglichen, dass genügend gesundes Gewebe vorhanden ist, das eine Tumorresektion ohne die Gefahr eines Leberversagens ermöglicht.

Deutlich weiter ist die Forschung in Bezug auf die PHD-Inhibitoren bei der Regeneration blutbildender Zellen. Im Tiermodell wurde beschrieben, dass diese Substanzen zur Blutbildung beitragen [39]. Später stellte man fest, dass deren orale Einnahme beim Menschen die Erythropoese zuverlässig unterstützt [40]. Aktuell werden diese Substanzen in Phase III Studien bei chronischer Niereninsuffizienz getestet um die begleitende Anämie zu bekämpfen[40]. Bei den Substanzen handelt es sich um PHD2-Inhibitoren, die dosisabhängig zu einer Stabilisierung von HIF1 $\alpha$ und HIF2 $\alpha$ führen [41].

Trotz der Tatsache, dass wir Sauerstoffmangel in der Regel mit etwas Negativem assoziieren, bieten möglicherweise gerade diese hochkonservierten Signalwege in der Wundheilung und der Regenerationsforschung bei selektivem Einsatz eine vielversprechende Therapiemöglichkeit. 


\section{Abkürzungen}

ATP: Adenosin-Triphosphat

GLUT1: Glucosetransporter 1

HIF: Hypoxie-induzierbarer Faktor

HRE: Hypoxia Response Element

iNOS: induzierbare NO-Synthetase

PHD: Prolylhydroxylase

VHL: Von Hippel Lindau

VEGF: Vascular endothelial growth factor

VEGF-R: Vascular endothelial growth receptor 


\section{Figurenlegende}

Figur 1: Der HIF-Pathway und seine wichtigsten Auswirkungen, Figur adaptiert und modifiziert nach Selvaraju V. et al.[42]. Pathway unter normoxischen Bedingungen (in blau). Die Prolylhydroxylase (PHD) hydroxyliert 2 Prolinreste an der $\alpha$-Untereinheit des Hypoxieinduzierbaren Faktors (HIF $\alpha$ ). Durch diese Hydroxylierung kann das von Hippel Lindau Protein (VHL) an HIF $\alpha$ binden, es wird ubiquitiniert und anschliessend proteasomal abgebaut. Unter hypoxischen Bedingungen (in schwarz) bleibt der Hydroxylierungsschritt aus, HIF kann als Heterodimer im Zellkern an das Hypoxia Response Element (HRE) in der Promotorregion seiner Zielgene binden und diese aktivieren. 


\section{Referenzen}

1. Field J, Fuhrman FA, Martin AW: Effect of temperature on the oxygen consumption of brain tissue. J Neurophysiol 1944, 7(2):117-126.

2. Bing RJ, Hammond MM, et al.: The measurement of coronary blood flow, oxygen consumption, and efficiency of the left ventricle in man. Am Heart J 1949, 38(1):124.

3. Fredholm BB, Linde B, Prewitt RL, Johnson PC: Oxygen-Uptake and Tissue OxygenTension during Adrenergic-Stimulation in Canine Subcutaneous Adipose-Tissue. Acta Physiol Scand 1976, 97(1):48-59.

4. Ziello JE, Jovin IS, Huang Y: Hypoxia-Inducible Factor (HIF)-1 regulatory pathway and its potential for therapeutic intervention in malignancy and ischemia. Yale J Biol Med 2007, 80(2):51-60.

5. Jun JC, Rathore A, Younas H, Gilkes D, Polotsky VY: Hypoxia-Inducible Factors and Cancer. Curr Sleep Med Rep 2017, 3(1):1-10.

6. Harwood SM, Yaqoob MM, Allen DA: Caspase and calpain function in cell death: bridging the gap between apoptosis and necrosis. Ann Clin Biochem 2005, 42:415431.

7. Yuan J: Neuroprotective strategies targeting apoptotic and necrotic cell death for stroke. Apoptosis 2009, 14(4):469-477.

8. Krijnen PA, Nijmeijer R, Meijer CJ, Visser CA, Hack CE, Niessen HW: Apoptosis in myocardial ischaemia and infarction. J Clin Pathol 2002, 55(11):801-811.

9. Schofield CJ, Ratcliffe PJ: Oxygen sensing by HIF hydroxylases. Nat Rev Mol Cell Biol 2004, 5(5):343-354.

10. Gorres KL, Raines RT: Prolyl 4-hydroxylase. Crit Rev Biochem Mol 2010, 45(2):106124.

11. Klotzsche-von Ameln A, Prade I, Grosser M, Kettelhake A, Rezaei M, Chavakis T, Flamme I, Wielockx B, Breier G: PHD4 Stimulates Tumor Angiogenesis in Osteosarcoma Cells via TGF-alpha. Mol Cancer Res 2013, 11(11):1337-1348.

12. Bruick RK, McKnight SL: A conserved family of prolyl-4-hydroxylases that modify HIF. Science 2001, 294(5545):1337-1340.

13. Jaakkola P, Mole DR, Tian YM, Wilson MI, Gielbert J, Gaskell SJ, von Kriegsheim A, Hebestreit HF, Mukherji M, Schofield CJ et al: Targeting of HIF-alpha to the von Hippel-Lindau ubiquitylation complex by 0-2-regulated prolyl hydroxylation. Science 2001, 292(5516):468-472.

14. Ratcliffe PJ: HIF-1 and HIF-2: working alone or together in hypoxia? J Clin Invest 2007, 117(4):862-865.

15. Stroka DM, Burkhardt T, Desbaillets I, Wenger RH, Neil DAH, Bauer C, Gassmann M, Candinas D: HIF-1 is expressed in normoxic tissue and displays an organ-specific regulation under systemic hypoxia. Faseb J 2001, 15(13):2445-2453.

16. Vaupel P, Harrison L: Tumor hypoxia: Causative factors, compensatory mechanisms, and cellular response. Oncologist 2004, 9:4-9.

17. Goda N, Ryan HE, Khadivi B, McNulty W, Rickert RC, Johnson RS: Hypoxia-inducible factor 1 alpha is essential for cell cycle arrest during hypoxia. Mol Cell Biol 2003, 23(1):359-369.

18. Raval RR, Lau KW, Tran MGB, Sowter HM, Mandriota SJ, Li JL, Pugh CW, Maxwell PH, Harris AL, Ratcliffe PJ: Contrasting properties of hypoxia-inducible factor 1 (HIF-1) 
and HIF-2 in von Hippel-Lindau-associated renal cell carcinoma. Mol Cell Biol 2005, 25(13):5675-5686.

19. Semenza GL, Nejfelt MK, Chi SM, Antonarakis SE: Hypoxia-Inducible Nuclear Factors Bind to an Enhancer Element Located 3' to the Human Erythropoietin Gene. P Natl Acad Sci USA 1991, 88(13):5680-5684.

20. Semenza GL, Wang GL: A Nuclear Factor Induced by Hypoxia Via Denovo ProteinSynthesis Binds to the Human Erythropoietin Gene Enhancer at a Site Required for Transcriptional Activation. Mol Cell Biol 1992, 12(12):5447-5454.

21. Jelkmann W: Erythropoietin: structure, control of production, and function. Physiol Rev 1992, 72(2):449-489.

22. Pugh CW, Ratcliffe PJ: Regulation of angiogenesis by hypoxia: role of the HIF system. Nat Med 2003, 9(6):677-684.

23. Krock BL, Skuli N, Simon MC: Hypoxia-induced angiogenesis: good and evil. Genes Cancer 2011, 2(12):1117-1133.

24. Liao D, Johnson RS: Hypoxia: a key regulator of angiogenesis in cancer. Cancer Metastasis Rev 2007, 26(2):281-290.

25. Shaw RJ: Glucose metabolism and cancer. Curr Opin Cell Biol 2006, 18(6):598-608.

26. Kaluz S, Kaluzova M, Liao SY, Lerman M, Stanbridge EJ: Transcriptional control of the tumor- and hypoxia-marker carbonic anhydrase 9: A one transcription factor (HIF-1) show? Bba-Rev Cancer 2009, 1795(2):162-172.

27. Swietach $\mathrm{P}$, Vaughan-Jones RD, Harris AL: Regulation of tumor $\mathbf{p H}$ and the role of carbonic anhydrase 9. Cancer Metast Rev 2007, 26(2):299-310.

28. Murry CE, Jennings RB, Reimer KA: Preconditioning with ischemia: a delay of lethal cell injury in ischemic myocardium. Circulation 1986, 74(5):1124-1136.

29. Zhao $\mathrm{H}$ : Ischemic postconditioning as a novel avenue to protect against brain injury after stroke. J Cerebr Blood F Met 2009, 29(5):873-885.

30. Thielmann M, Kottenberg E, Kleinbongard P, Wendt D, Gedik N, Pasa S, Price V, Tsagakis K, Neuhauser M, Peters J et al: Cardioprotective and prognostic effects of remote ischaemic preconditioning in patients undergoing coronary artery bypass surgery: a single-centre randomised, double-blind, controlled trial. Lancet 2013, 382(9892):597-604.

31. Speechlydick ME, Mocanu MM, Yellon DM: Protein-Kinase-C - Its Role in Ischemic Preconditioning in the Rat. Circ Res 1994, 75(3):586-590.

32. Ytrehus K, Liu YG, Downey JM: Preconditioning Protects Ischemic Rabbit Heart by Protein-Kinase-C Activation. Am J Physiol 1994, 266(3):H1145-H1152.

33. de la Barca JMC, Bakhta O, Kalakech H, Simard G, Tamareille S, Catros V, Callebert J, Gadras C, Tessier L, Reynier P et al: Metabolic Signature of Remote Ischemic Preconditioning Involving a Cocktail of Amino Acids and Biogenic Amines. J Am Heart Assoc 2016, 5(9).

34. Maes C, Carmeliet G, Schipani E: Hypoxia-driven pathways in bone development, regeneration and disease. Nat Rev Rheumatol 2012, 8(6):358-366.

35. Scheid A, Wenger RH, Christina H, Camenisch I, Ferenc A, Stauffer UG, Gassmann M, Meuli $M$ : Hypoxia-regulated gene expression in fetal wound regeneration and adult wound repair. Pediatr Surg Int 2000, 16(4):232-236.

36. Tandara AA, Mustoe TA: Oxygen in wound healing - More than a nutrient. World J Surg 2004, 28(3):294-300.

37. Michalopoulos GK: Liver regeneration after partial hepatectomy: critical analysis of mechanistic dilemmas. Am J Pathol 2010, 176(1):2-13. 
38. Schadde E, Tsatsaris C, Swiderska-Syn M, Breitenstein S, Urner M, Schimmer R, Booy C, Z'graggen BR, Wenger RH, Spahn DR et al: Hypoxia of the growing liver accelerates regeneration. Surgery 2017, 161(3):666-679.

39. Forristal CE, Winkler IG, Nowlan B, Barbier V, Walkinshaw G, Levesque JP:

Pharmacologic stabilization of HIF-1 alpha increases hematopoietic stem cell quiescence in vivo and accelerates blood recovery after severe irradiation. Blood 2013, 121(5):759-769.

40. Provenzano R, Besarab A, Sun CH, Diamond SA, Durham JH, Cangiano JL, Aiello JR, Novak JE, Lee T, Leong R et al: Oral Hypoxia-Inducible Factor Prolyl Hydroxylase Inhibitor Roxadustat (FG-4592) for the Treatment of Anemia in Patients with CKD. Clin J Am Soc Nephro 2016, 11(6):982-991.

41. Yeh TL, Leissing TM, Abboud MI, Thinnes CC, Atasoylu O, Holt-Martyn JP, Zhang D, Tumber A, Lippl K, Lohans CT et al: Molecular and cellular mechanisms of HIF prolyl hydroxylase inhibitors in clinical trials. Chem Sci 2017, 8(11):7651-7668.

42. Selvaraju V, Parinandi NL, Adluri RS, Goldman JW, Hussain N, Sanchez JA, Maulik N: Molecular Mechanisms of Action and Therapeutic Uses of Pharmacological Inhibitors of HIF-Prolyl 4-Hydroxylases for Treatment of Ischemic Diseases. Antioxid Redox Sign 2014, 20(16):2631-2665. 